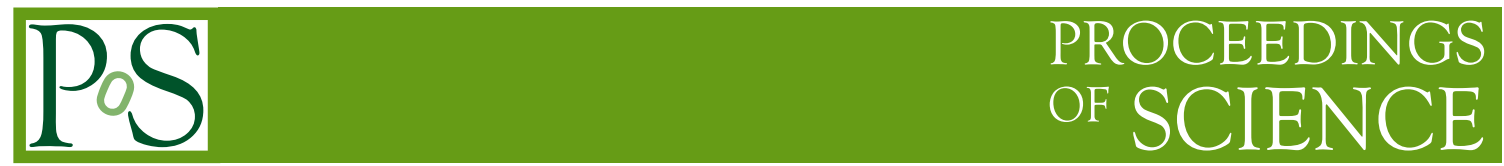

\title{
Hadron Spectroscopy and Resonances : Review
}

\section{Padmanath*}

Institüt für Theoretische Physik, Universität Regensburg, 93053 Regensburg, Germany

E-mail: Padmanath. M@physik.uni-regensburg.de

I review recent results on hadron spectroscopy using lattice QCD. In light of the discoveries in heavy baryon sector at $\mathrm{LHCb}$ over the past few years, lattice calculations in this regard are emphasized. Investigations on light baryon, heavy-heavy and heavy-light meson resonances are also discussed.

The 36th Annual International Symposium on Lattice Field Theory - LATTICE2018 22-28 July, 2018

Michigan State University, East Lansing, Michigan, USA.

${ }^{*}$ Speaker. 


\section{Introduction}

The observation of many interesting resonance structures in hadronic final states in recent years has spurred huge interest in hadron spectroscopy investigations both theoretically and experimentally. The discovery of a doubly charmed baryon $\Xi_{c c}^{+}(c c u)$ with a mass of $3621.40 \pm 0.78 \mathrm{MeV}$ by the LHCb Collaboration [1] marks an important milestone in our quest to understand Quantum Chromo Dynamics (QCD), the theory of strongly interacting hadrons. Another highlight is the unambiguous observation by the LHCb Collaboration of five new narrow $\Omega_{c}$ resonances in $\Xi_{c}^{+} K^{-}$ invariant mass distribution in the energy range between $3000-3120 \mathrm{MeV}$ [2]. Four out of these five resonances have been later confirmed by the Belle Collaboration [3]. Compelling evidence for charged meson-like resonances in the heavy quarkonium energy range indicate the existence of four quark bound states (for review see Refs. $[4,5,6]$ ). Similarly, the LHCb discovery of exotic structures in the $J / \psi p$ channel confirms the existence of charmonium-nucleon pentaquark resonances [7]. Measuring the properties of these resonances can further enhance our understanding of QCD. Experiments are engaged in search for new resonances with an aim of precisely measuring their mass, lifetime, production and decay mechanisms, etc. [8, 9, 10]. This motivates theoretical investigations to make predictions for such resonances, that can be put to test in various experiments such as LHCb, Belle.

Lattice QCD calculations of hadron spectroscopy have achieved remarkable progress over the past ten years in making large volume simulations with physical quark masses, impressive statistical precision and good control over different systematic uncertainties [11, 12, 13, 14, 15, 16]. Several lattice groups have been performing detailed systematic investigations of stable hadrons that are well below the lowest allowed strong decay threshold. A discussion of various lattice systematic uncertainties and how they are addressed by different lattice groups is made in Ref. [17]. Multiple exploratory studies are also being performed in order to understand and to help interpreting these observed hadrons close to strong decay thresholds and resonances above the threshold. A detailed review on methodologies for treating the hadronic resonances on the lattice and various lattice calculations along these lines can be found in Ref. [18].

In this report, I review the results from lattice calculations that are of particular relevance considering the present and future experimental progress. In Section 2, I briefly outline the basic lattice methodology that is relevant for the results presented in this review. In Section 3, I summarize the high precision lattice results for masses of stable hadrons. In Section 4, I discuss the studies of excited hadrons that have been in the scientific limelight considering recent experimental discoveries. In Sections 5 and 6, I review the recent lattice calculations of hadronic resonances involving rigorous finite volume analysis. In Section 7, I discuss recent lattice results on unconventional hadrons that do not fit into $\bar{q} q$ and $q q q$ picture, and summarize the review in Section 8.

\section{Lattice methodology}

The physics of hadrons is commonly extracted on the lattice from the finite volume Euclidean correlation functions. In order to study the hadron spectrum, one computes the Euclidean two point 
correlation functions,

$$
C_{i j}\left(t_{f}-t^{\prime}\right)=\left\langle O_{i}\left(t_{f}\right) O_{j}^{\dagger}\left(t^{\prime}\right)\right\rangle=\sum_{n} \frac{Z_{i}^{n} Z_{j}^{n *}}{2 E_{n}} e^{-E_{n}\left(t_{f}-t^{\prime}\right)},
$$

between hadronic currents $\left(O_{i}(t)\right)$ that are built to respect the quantum numbers of interest. The operator $O_{i}(t)$ can couple to all the states, including single-particle levels as well as multi-particle levels and their radial excitations, with these quantum numbers. A general practice to extract the excited spectrum is to compute matrices of correlation functions between a basis of interpolators $O_{i}(t)[19,20,21,22]$ and to solve the Generalized EigenValue Problem (GEVP) [23, 24, 25]

$$
C_{i j}(t) v_{j}^{n}\left(t-t_{0}\right)=\lambda^{n}\left(t-t_{0}\right) C_{i j}\left(t_{0}\right) v_{j}^{n}\left(t-t_{0}\right)
$$

Energies $\left(E_{n}\right)$ are extracted from exponential fits to the large time behavior of the eigenvalues $\lambda^{n}\left(t-t_{0}\right)$. The operator state overlaps $Z_{i}^{n}=\left\langle O_{i} \mid n\right\rangle$ are related to the eigenvectors $v_{j}^{n}\left(t-t_{0}\right)$.

Hadron masses that are well below the lowest allowed strong decay threshold and hence deeply bound are trivially related to the lattice energies $m_{H}=E_{\text {lat }}(\mathbf{p}=\mathbf{0})$ up to exponentially suppressed corrections. Lattice investigations of these stable hadrons require only an extraction of ground states. Simple fits to the large time behavior of single correlation functions provide quite precise results. This has been in practice, since the early applications of lattice QCD in hadron spectroscopy. There is a number of ground state baryons that are stable to strong decays and can be studied quite precisely on the lattice.

Most hadrons appear above or close to strong decay threshold. There is no direct procedure to extract the energies for these hadrons from the discrete spectrum on the lattice [26]. Properties of these near or above threshold hadron excitations have to be inferred from the infinite volume scattering matrices. A widely used approach to extract the infinite volume scattering matrix from the discrete finite volume energy spectrum is á la Lüscher [27, 28, 29]. This approach relates the infinite volume phase shifts, that possess all information on the scattering process, to the discrete energy spectrum in the finite volume through known kinematic functions. Several extensions and generalizations to Lüscher's original proposal have been made over the past five years: e.g. to describe the two particle scattering with different particle identities, in different boundary conditions, in moving frames, multiple partial waves, coupled channel scattering and more (see Refs. [30, 31]). Updates on efforts to build extensions that relate discrete finite volume energy spectrum to threebody scattering amplitudes have been reported in this conference [32, 33]. A detailed discussion on the formalism, its extensions and a comprehensive list of references can be found in Ref. [18].

There exist other formalisms to extract infinite volume scattering information from the lattice such as the HALQCD method [34, 35], finite volume Hamiltonian EFT [36] and the relatively new optical potential method [37]. Attempts to relate the three-body scattering amplitudes with the discrete energy spectrum in the finite volume are also being made [38, 39, 40, 41]. For detailed information on these formulations the reader may refer to the original articles, while a summary of lattice investigations following these approaches can be found in the reviews from previous lattice conferences [17, 42].

Most of the results using a finite volume analysis presented in this review are based on Lüscher's formalism and its extensions. Novel lattice QCD techniques such as distillation [43], stochastic 
distillation [44] and unbiased noise reduction techniques [45] to compute the all-to-all quark propagation diagrams has made lattice calculations using up to two hadron interpolators and involving rigorous finite volume analysis possible. Lattice studies of resonances discussed in this review are performed using such techniques and consider all required Wick contractions, except those with OZI suppressed heavy quark self-annihilation diagrams, where applicable, to compute the correlation matrices. There is, as yet, no numerical study of hadronic resonances using three hadron interpolators, except for investigations of nuclear binding energies.

\section{Ground state hadrons}

Postdicting the mass of ground state hadrons is a standard benchmark procedure that boosts confidence in our methodology and hence lends support to predictions. Several calculations have been performed with good control over the statistical as well as systematic uncertainties and have precisely predicted/postdicted meson ground state masses (c.f. Ref. [14]). Most of these have also been discussed in previous lattice conferences [42]. In what follows, I focus on the recent high precision lattice results on the light as well as heavy ground state baryons.
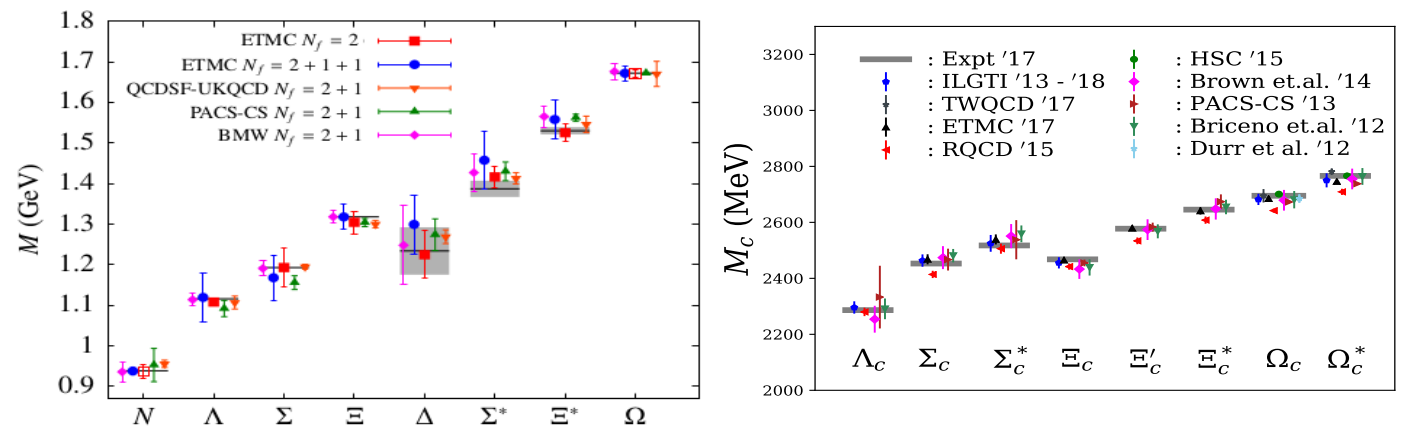

Figure 1: Left : (figure adapted from Ref. [46]) Lattice results for the octet and decuplet light baryon masses. The horizontal lines represent the experimental masses and the bands represent the widths. Unfilled symbols indicate baryon masses that have been used as inputs to the calculation, whereas the filled symbols refer to postdictions. Right : Lattice results for single charmed baryon masses.

Light and strange baryons : The left of Fig. 1 is a summary plot of lattice results for the octet and the decuplet light baryon masses at physical pion mass. The results from "BMW $N_{f}=2+1$ " [12] and "ETMC $N_{f}=2+1+1$ " [47] are chiral and continuum extrapolated, whereas the results from "ETMC $N_{f}=2$ " [46], "PACS-CS $N_{f}=2+1$ " [48] and "QCDSF-UKQCD $N_{f}=2+1$ " [49] are at physical pion mass, but with no continuum extrapolations. Note that the details of the methodology considerably differ between different lattice calculations: e.g. the lattice ensembles being used, the fermion and the gauge field actions, the degree of control over the lattice systematics (e.g. arising from chiral and continuum limits), etc. The success of lattice investigations are reflected in the mutual agreement between their results and their agreement with experiments. One of the most interesting investigations of recent times is the precise estimation of the energy splittings in $N, \Sigma, \Xi, D$ and $\Xi_{c c}$ isospin multiplets from lattice QCD and QED computations with $N_{f}=1+1+1+1$ fermions by BMW collaboration [16]. This was discussed in a plenary talk at Lattice 2014 [50]. 
Several other exploratory lattice calculations have also been performed to estimate the light as well as strange baryons (e.g. Refs. [51, 52, 53, 54]). Note that even in the absence a good chiral and continuum extrapolations, the results from these calculations make precise predictions for quantum numbers of the ground state light and strange baryon masses, e.g. for the recently discovered $\Omega^{*-}$ baryon by the Belle Collaboration [8].

Singly charmed baryons : In the right of Fig. 1, I present a summary of recent lattice results for the masses of singly charmed baryons. The experimental masses are shown as gray horizontal lines. The results from "ILGTI '13-'18" [55, 56, 57, 58], "Briceno et.al. '12" [59] and "Brown et. al. '14" [60] are based on mixed action calculations and are chiral and continuum extrapolated to the physical limits. "PACS-CS '13" [15] and "ETMC '17" [46] refer to the results calculated at physical pion mass, whereas "RQCD '15" [61] refers to chiral extrapolated results, all at a single lattice spacing. "TWQCD '17" [62] and "Dürr et. al. "12" [63] are exploratory investigations on single lattice QCD ensembles at heavier than physical pion masses. "HSC " 15 " refers to the results from an exploratory study of excited charm baryon spectrum on an anisotropic ensemble with $m_{\pi}=391 \mathrm{MeV}$ [64]. It is very evident from the figure that there is good overall agreement between all the lattice estimates and also with the respective experimental masses. Note that lattice results for heavy quarks are expected to be severely affected by discretization effects. Hadrons with larger number of valence heavy quarks are expected to be affected with larger discretization effects. Some of these calculations utilize novel techniques like Fermilab approach to tune the heavy quark masses, and mass differences and dimensionless mass ratios to perform extrapolations. These procedures are expected to remove the leading discretization effects and to provide good control in continuum extrapolations. In this sense, the agreement between different lattice estimates and with experiment imply small discretization effects in comparison with the statistical uncertainties for the lattice fermion actions used for charm quarks at the lattice spacings utilized. This also gives confidence in making robust and reliable predictions for the doubly and the triply heavy baryons.
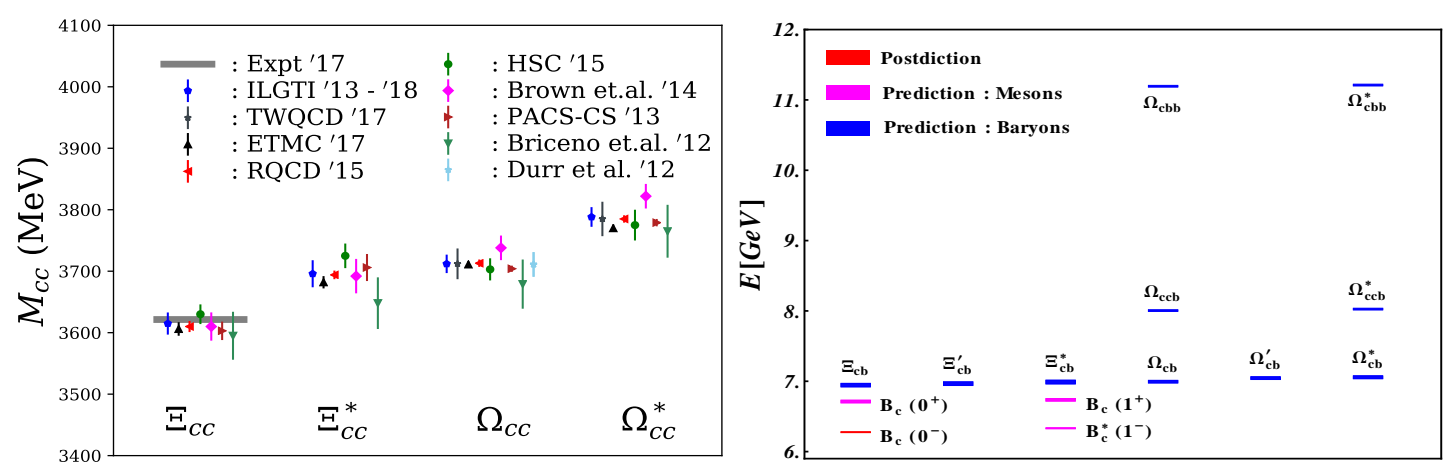

Figure 2: Left : Lattice predictions for the masses of the doubly charm baryons. The experimental mass for $\Xi_{c c}\left(1 / 2^{+}\right)$as determined by LHCb [9] is shown as horizontal band. Right : Lattice estimates for charmedbottom hadron masses as determined in Ref. [65].

Doubly charmed baryons : In the left of Fig. 2, I present lattice predictions for the ground state doubly charmed baryon masses. It is evident from the figure that these estimates for the only known doubly heavy system $\Xi_{c c}\left(1 / 2^{+}\right)$show good agreement with each other. They are all consistent with the experimental mass as determined by the LHCb Collaboration [9]. Note that all these lattice estimates predate the LHCb discovery and hence were predictions for this state. 
This clearly demonstrates the potential of lattice QCD techniques to make reliable predictions in the heavy baryon sector ${ }^{1}$. Not all the results have been estimated after a continuum extrapolation. This indicates that the cut-off uncertainties, that are expected to be severe in the heavy hadron observables, are small. In this figure, I have omitted the results from a few early quenched lattice calculations $[66,67,68]$ and a dynamical calculation [69] on the ground state heavy baryon masses, which are also in good agreement with results presented in Fig. 2.

All the lattice predictions for the mass of $\Xi_{c c}\left(1 / 2^{+}\right)$, shown in Fig. 2(left), lie $\sim 100 \mathrm{MeV}$ above the SELEX measurement for the mass of a doubly charmed baryon (3519(1) MeV) [70]. As pointed out earlier, a precision determination of the energy splittings in $N$ and $\Xi_{c c}$ isospin multiplets from lattice QCD and QED computations with $N_{f}=1+1+1+1$ fermions was performed by BMW collaboration [16]. In this calculation, they postdict the neutron-proton mass splittings with an accuracy of $0.3 \mathrm{MeV}$. The lattice prediction for the energy splitting between the isospin partners of $\Xi_{c c}\left(1 / 2^{+}\right)$from this calculation is $2.16(11)(17) \mathrm{MeV}$. This excludes the possibility that the SELEX measured doubly charmed baryon candidate to be the isospin partner of $\Xi_{c c}^{+}(3621.4 \mathrm{MeV})$.

Charmed bottom baryons : In the right of Fig. 2, I present the lattice estimates for the masses of hadrons with at least one charm and one bottom quark from mixed action calculations using overlap fermions for quark masses up to charm and a non-relativistic QCD formulation for bottom studied on $N_{f}=2+1+1$ HISQ fermion MILC ensembles [65]. This investigation was carried out on three ensembles with different lattice spacings to achieve good control over the discretization effects. Utilizing the energy splittings and dimensionless mass ratios, the authors perform controlled chiral and continuum extrapolations to obtain reliable predictions for many yet to be discovered charmed-bottom hadrons. The lattice postdiction for the only discovered charmedbottom hadron $B_{c}$ meson is found to be in good agreement with the experimental mass. Note that the experimental mass of this $B_{c}$ meson was originally found to be in agreement with the lattice prediction [14]. The predcitions in Fig. 2(right) for other ground state $B_{c}$ meson masses are also in agreement with the predictions in Ref. [14]. The mass estimates for charmed-bottom baryons are also found to be in good agreement with the only existing previous dynamical calculation [60] performed on RBC-UKQCD ensembles with two different lattice spacings.

All the results presented above are estimated within the single hadron approach, where only three quark interpolators are considered in the analysis and the effects of any nearby strong decay thresholds are neglected. This is justified for most baryons discussed above, considering the fact that they are deeply below the respective lowest strong decay thresholds. However, this approach is questionable for baryons like $\Delta, \Sigma^{*}, \Xi^{*}$, etc., which are resonances and can decay into one or more strong decay modes. Hence attributing lattice energy levels to the resonance energies is not appropriate and they require a rigorous finite volume analysis as mentioned in Section 2. Recent lattice calculations in this regard will be part of the discussion in Sections 5 and 6.

\section{Excited hadron spectroscopy}

The first step in performing a rigorous finite volume treatment on the lattice is to reliably extract the discrete energy spectrum. As mentioned previously, a standard practice these days is to

\footnotetext{
${ }^{1}$ Several successful lattice predictions exist in the heavy meson sector (c.f. Ref. [13, 14]).
} 
evaluate correlation matrices (Eq. 2.1) for a basis of interpolating operators and solve the GEVP (Eq. 2.2) to extract the excited state information. A procedure that is developed and followed by HSC to build meson interpolator basis on the lattice have been quite successful in extracting multiple excited states and reliably identify their quantum numbers [71, 72, 21, 22]. This procedure has been utilized extensively by HSC in their lattice investigations for light mesons, charmed mesons and charmonia [73, 22, 74, 75, 76]. More recently RQCD collaboration has also started practicing this formulation to study the charmonium spectrum [77]. Lattice calculations utilizing other interpolator bases in extracting excited state spectrum for heavy quarkonium as well as heavy light mesons also exist in the literature $[78,79,80]$.

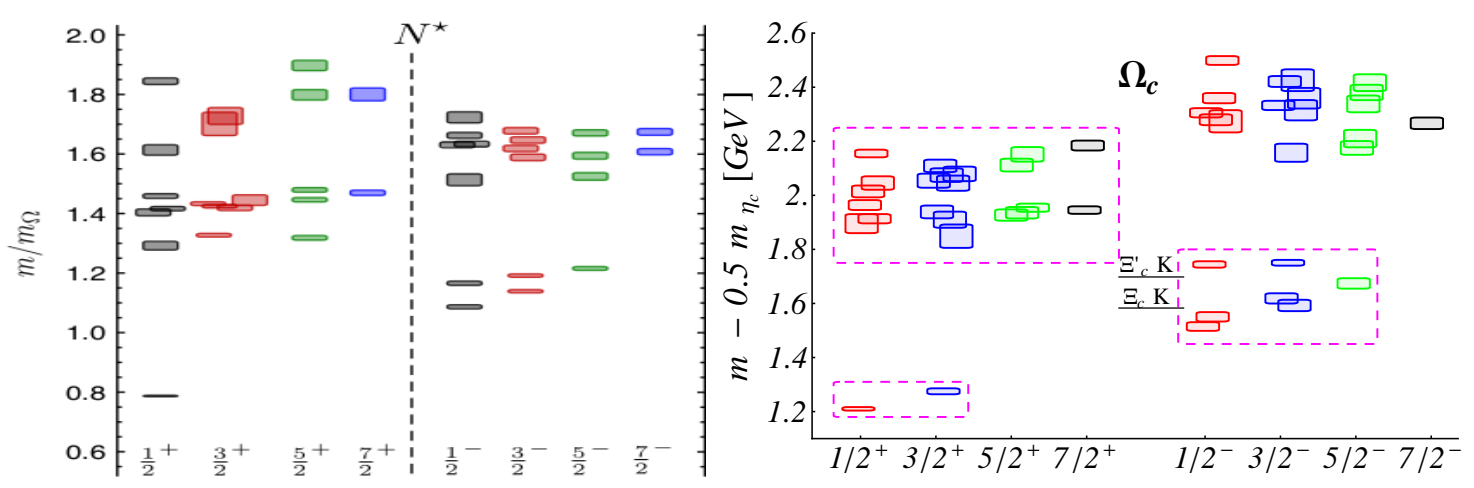

Figure 3: Spin identified excited state spectra of nucleon (left) and $\Omega_{c}$ baryon (right) as determined in Refs. [52] and [81], respectively.

Excited baryons : An equivalent procedure to systematically build baryon interpolator basis have been developed by the LHP Collaboration more than a decade ago [19, 20]. Early calculations following these interpolators have also been reported in Refs. [82]. Over the past years, HSC has realized these interpolators and studied light and strange baryons [51, 52, 53] as well as charm baryons with one, two and three valence charm quarks [83, 84, 64, 81]. Fig. 3 shows the spin identified excited spectra of the nucleon (left) and $\Omega_{c}$ baryon (right) as determined in Refs. [52] and [81], respectively. Similar lattice investigation to extract triply bottom baryons was reported in Ref. [85].

All lattice calculations discussed in this section follow the single hadron approach and assume that the lattice estimates for the mass of resonances are correct up to the respective decay width. This approach is justified in determining the energy of excitations that are well below the lowest allowed strong decay threshold. Narrow elastic resonances can also be approximately studied within this approach. Most light hadron resonances have decay widths of the order of $100 \mathrm{MeV}$ and hence are not appropriate to be studied in this way. However narrow resonances, such as the recently discovered excited $\Omega_{c}$ baryons by the LHCb Collaboration [2], can be studied using this approach. In what follows, we discuss such a lattice calculation that made precise predictions for the masses and quantum numbers of these excited $\Omega_{c}$ baryons.

Excited $\Omega_{c}$ baryons : In the left of Fig. 4, I present the event distribution in the $\Xi_{c}^{+} K^{-}$decay channel displaying the five narrow peaks discovered and interpreted as excited $\Omega_{c}$ baryons by the LHCb Collaboration [2]. The resonance structures can be seen to be quite narrow $(\lesssim 10$ $\mathrm{MeV}$ ). Four out of these five resonances have been later confirmed using $e^{+} e^{-}$collision data from 

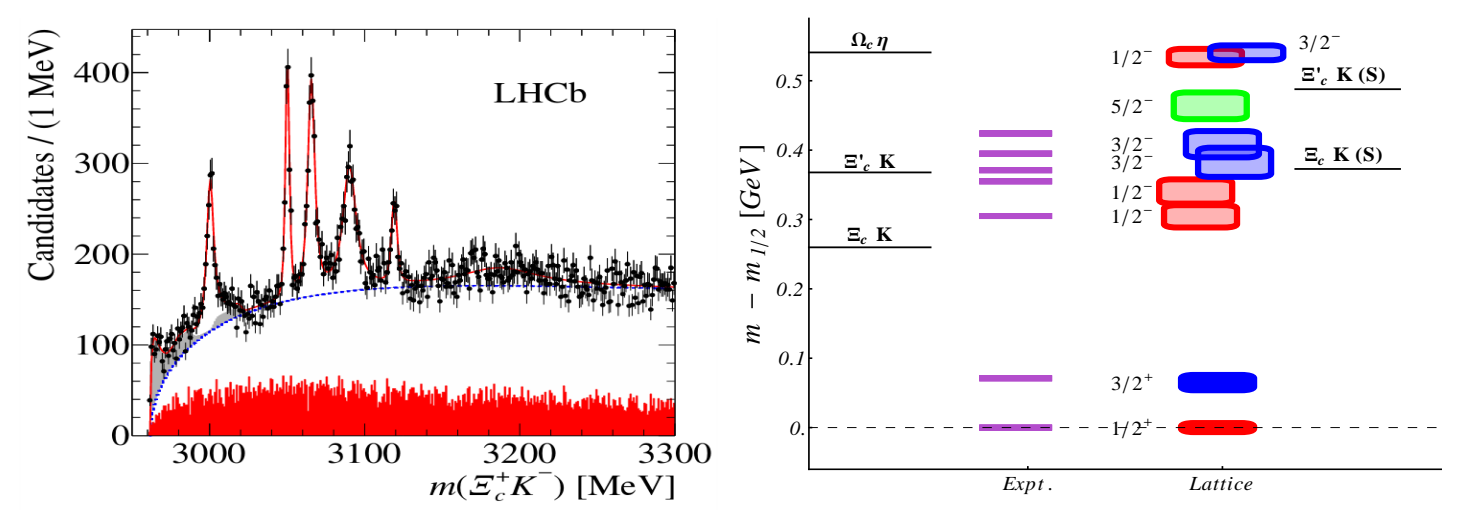

Figure 4: Left : (Figure adapted from Ref. [2]) The resonance structures observed in the $\Xi_{c}^{+} K^{-}$decay mode interpreted as excited $\Omega_{c}$ baryons by the LHCb Collaboration. Right : Comparison of experimental masses of $\Omega_{c}$ baryons with the excited energy spectra on the lattice and their quantum number assignments. Nearby scattering thresholds are shown on the left and the relevant non-interacting level positions on the lattice are shown on the right as black horizontal lines.

Belle [3]. In the right of Fig. 4, I present a comparison of the masses of the seven experimentally known $\Omega_{c}$ baryons (indicated by the horizontal magenta lines) with the lowest nine $\Omega_{c}$ baryons as extracted from a lattice calculation [81] within the single hadron approach on a $N_{f}=2+1$ anisotropic clover ensemble with a pion mass of $391 \mathrm{MeV}$ and a physical spatial volume $(1.9 \mathrm{fm})^{3}$ generated by HSC. The results from this calculation correctly postdict the mass of $\Omega_{c}\left(1 / 2^{+}\right)$baryon and the hyperfine splitting in the ground state $\Omega_{c}$ baryons $\left(E_{3 / 2^{+}}-E_{1 / 2^{+}}\right)$. Note that the hyperfine splittings are generally observed to be quite sensitive to discretization uncertainties and the agreement between the lattice and experiment indicates such uncertainties on $\Omega_{c}$ baryons in this lattice setup are small. Lattice also predicts five states as shown in the figure, with quantum numbers " $1 / 2^{-}, 1 / 2^{-}, 3 / 2^{-}, 3 / 2^{-}$and $5 / 2^{-}$" in the region of experimental discovery. Note that these results were reported in Lattice 2014 [86] as well as in Charm 2013, 2015 [87, 64] and hence predate the LHCb discovery. An immediate extension to this calculation would be to include interpolators that are related to the nearby non-interacting baryon-meson levels and to perform a finite volume analysis. Such calculations including baryon-meson interpolators in the analysis are in their early stage of development and will be discussed in Section 6. More lattice calculations of the excited baryon spectrum will be highly appreciated by the scientific community, anticipating the discovery of many more baryons in experiments like LHCb and Belle.

\section{Meson resonances on the lattice}

The majority of hadrons are resonances and can decay via strong interactions. Most resonances have large decay widths $\mathscr{O}(100 \mathrm{MeV})$ and can decay into different sets of hadronic final states. Hence studies of hadronic resonances on the lattice demand a finite volume treatment. A good way to start such calculations is to explore the easiest cases of elastic resonances and shallow bound states. Gradually relaxing various simplifying approximations, one may investigate more complicated scenarios like the effects of inelastic thresholds, coupled channel scattering and so on. 
Elastic scattering in light mesons : The pseudoscalar-pseudoscalar elastic scattering in the light and strange meson sector are two widely performed benchmark calculations among different lattice groups. There have been many lattice investigations of the $\rho$ (vector) meson in $p$-wave $\pi \pi$ scattering $[88,89,90,91,92,93,94,95,96,97,98]$. Some recent calculations in this channel have also been reported during this meeting. The coupling $g_{\rho . \pi \pi}$ has been generally observed to be independent of the pion mass in the elastic regime. A summary of different lattice results is made in Fig. 12 of Ref. [98]. It is seen that a dimensionless ratio $m_{\rho} / m_{N}$ is found to roughly indicate linear dependence with the pion mass squared leading to the experimental value in the chiral limit. The results from $N_{f}=2$ calculations are found to be scattered around the results from $N_{f}=2+1$ studies. A discussion on the pion mass dependence and the quenching effects of strange sea on the mass of the $\rho$ meson can be found in Ref. [98]. The $K^{*}$ meson is also studied in many lattice calculations through $p$-wave $K \pi$ scattering [99, 100, 101, 95, 102]. Among the calculations cited above, two unique calculations are those performed by HSC a few years back [94, 100, 101]. In these articles, the authors have studied the effects of inelastic threshold by performing a coupled channel finite volume analysis.
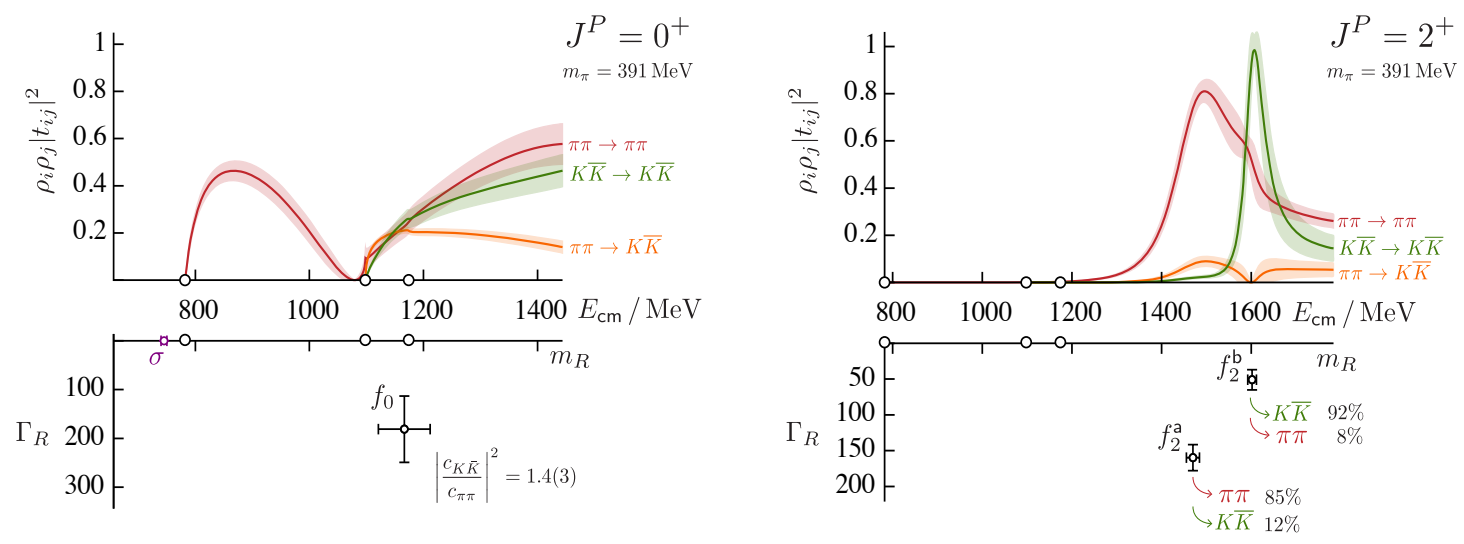

Figure 5: (Figure adapted from Ref. [103]) Coupled $\pi \pi-K \bar{K}$ amplitudes for isoscalar-scalar (left) and isoscalar-tensor (right) mesons. The three black circles in the real axis are the scattering thresholds corresponding to $\pi \pi, K \bar{K}$ and $\eta \eta$. In both the channels, $\eta \eta$ is found to be approximately decoupled. Pole singularities determining the features of the coupled channel scattering amplitudes are shown along with their uncertainties that include the variation in amplitude parameterizations.

Coupled channel scattering in light mesons : Recently HSC has performed a coupled channel $(\pi \pi, K \bar{K}, \eta \eta)$ investigation in the isoscalar-scalar channel, where the $\sigma$ meson and the $f_{0}(980)$ appear [103]. This calculation is an extension of their earlier investigation with $s$-wave $\pi \pi$ elastic scattering [104] and also includes an investigation of $d$-wave couple channel scattering. The summary of their results are as shown in Fig. 5. Owing to the heavy pion mass of $391 \mathrm{MeV}$, the $\sigma$ meson is a stable bound state in their setup. As in experiment, the $f_{0}(980)$ features as a dip in the $\pi \pi$ cross section close to the $K \bar{K}$ threshold. Two resonance peaks observed in the $d$-wave scattering amplitudes are argued to be related to $f_{2}(1270)$ and $f_{2}^{\prime}(1525)$, with the lighter peak decaying predominantly to $\pi \pi$ and the heavier peak to $K \bar{K}$. There also exist lattice efforts employing lighter pion masses to investigate this channel from HSC [105] and others [106]. In another recent arti- 
cle, HSC has demonstrated application of their techniques and the analysis of dynamically coupled partial waves in isospin-2 $\rho \pi$ scattering [107].
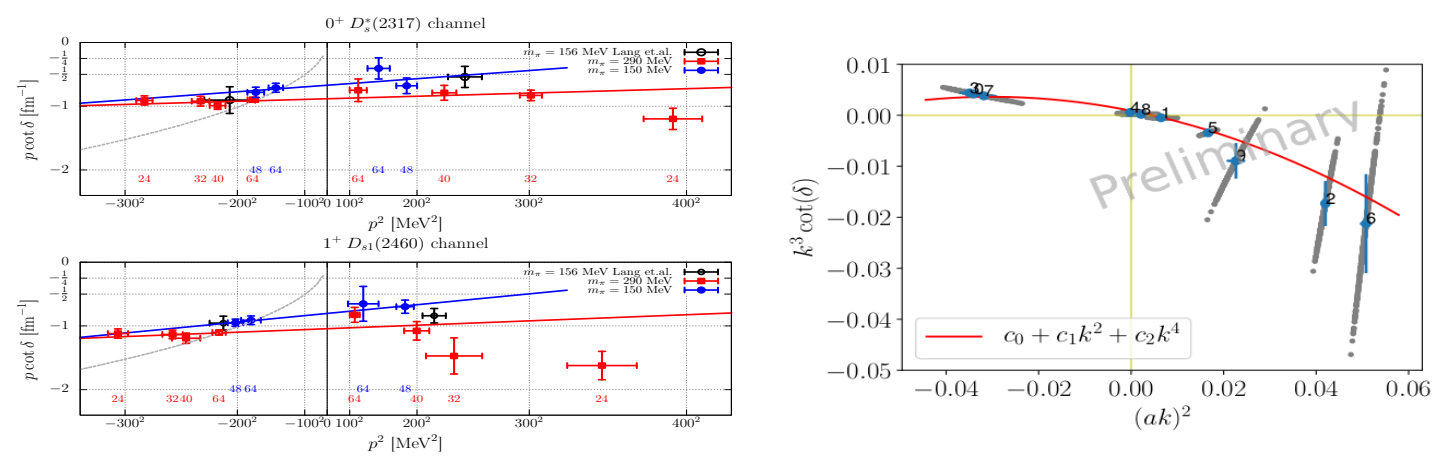

Figure 6: Left: (Figure adapted from Ref. [108]) $p \cot \delta$ as a function of $p^{2}$ for the scalar $\left(0^{+}\right.$, top) and vector $\left(1^{+}\right.$, bottom) heavy-strange mesons. The red and blue lines are linear fits to the data determining the bound state position from the intersection with the bound state constraint curve $\left(i p=-\sqrt{-p^{2}}\right.$ ) shown in gray dashed curves. Right: $k^{3} \cot \delta$ as a function of $k^{2}$ for vector $\left(1^{-}\right)$charmonium mesons from a calculation by the RQCD Collaboration. The red curve indicates a fit to the phase shift observed with a quartic fit form to describe a bound state and a resonance.

Charmed mesons : In contrast to phenomenological expectations, the scalar $0^{+}$and axialvector $1^{+}$ground states of heavy-strange mesons are found to be narrow and below the scattering thresholds $K D$ and $K D^{*}$, respectively. First calculation involving elastic scattering was performed in Ref. [109] on a PACS-CS ensemble with physical spatial volume $(2.9 \mathrm{fm})^{3}$ and near to physical pion mass. In a recent calculation by the RQCD Collaboration, the authors study these mesons utilizing six lattice QCD ensembles with $N_{f}=2$ non-perturbatively $\mathscr{O}(a)$ improved Wilson sea quarks at $a=0.07 \mathrm{fm}$, covering several spatial volumes with $L$ as large as $4.5 \mathrm{fm}$ and two different pion masses (290 MeV and $150 \mathrm{MeV}$ ). Performing a phase shift analysis and employing the effective range approximation, they determine the bound state masses and the coupling with the respective thresholds. In the left of Fig. 6, linear fits to the phase shift data in the scalar as well as the axialvector channel to describe the $D_{s 0}^{*}(2317)$ and the $D_{s 1}(2460)$ are shown. They also extract lattice levels in the axialvector channel related to the $D_{s 1}(2536)$ resonance, which are resolved sufficiently well using only the $\bar{c} s$ interpolators. The energies of these three states are found to be very sensitive to the pion mass (changes $\sim 30 \mathrm{MeV}$ from $290 \mathrm{MeV}$ to $150 \mathrm{MeV}$ ) in comparison with the changes in the energies for ground state pseudoscalar and vector $D_{s}$ mesons, which are 3 and $7 \mathrm{MeV}$, respectively. In addition to masses, they also determine the weak decay constants $f_{V}^{0^{+}}$and $f_{A}^{1^{+}}$of the $D_{s}$ mesons.

Excited charmonium : In the charmonium spectrum below $4 \mathrm{GeV}$, the vector channel has two bound states $J / \psi, \psi(2 S)$ and a resonance $\psi(3770)$, whereas in the scalar channel there is a bound state $\chi_{c 0}(1 P)$ and a recently discovered resonance $\chi_{c 0}(2 P)$ [110]. The scalar channel is interesting due to the presence of another candidate $X(3915)$, for which the quantum numbers are not yet known, but are expected to be either $0^{++}$or $2^{++}$. There has been only one previous calculation of these channels within the elastic scattering of $D \bar{D}$ performed in the rest frame [111]. Recent efforts by the RQCD Collaboration in studying the low lying resonant spectra in scalar and vector charmonia in the moving frames were reported at this meeting. Using two ensembles 
with $m_{\pi} \sim 280 \mathrm{MeV}$ and $m_{K} \sim 467 \mathrm{MeV}$, and with spatial extents $L \sim 2 \mathrm{fm}$ and $L \sim 2.7 \mathrm{fm}$, they investigate scattering amplitudes in the vector and scalar charmonium channels up to an energy of $4 \mathrm{GeV}$. In the right of Fig. 6, I present the phase shifts as a function of the momentum squared for the vector charmonium channel as determined by the RQCD Collaboration.

\section{Baryon resonances on the lattice}

In contrast to the meson sector, baryon resonances have received very little attention, largely due to the computational challenges. Evaluation of large number of Wick contractions possibly involving also annihilation diagrams, large computational and storage requirements owing to increase in the number of valence quarks and exponential degradation of signal-to-noise ratio demand humongous amount of computational resources. In addition to all these, the non-zero spin of baryons complicates the phase shift analysis. In the physical pion mass limit, more challenges appear with the opening of new scattering thresholds, including those involving three or more hadron scattering. Over the past two decades several lattice calculations have been performed of excited light as well as strange baryons employing three-quark interpolators and following a single hadron approach (see review Ref. [112]). Many of these calculations are quite remarkable considering the lattice technologies available then. New lattice technologies to compute all-to-all quark propagators $[43,44]$ and various theoretical developments following Lüscher's finite volume method promise precise determination of the finite volume spectrum and a reliable procedure to extract the resonance information therefrom. Following these procedures, there has been a calculation to determine the isospin-1/2 $N \pi$ scattering amplitude in the $s$-wave to describe the negative parity excitations $N(1535)$ and $N(1650)$ [113]. Below, I discuss two recent calculations investigating isospin-3/2 and $1 / 2 N \pi$ scattering in $p$-wave to describe the $\Delta$ baryon and the Roper resonance $(N(1440))$, respectively.
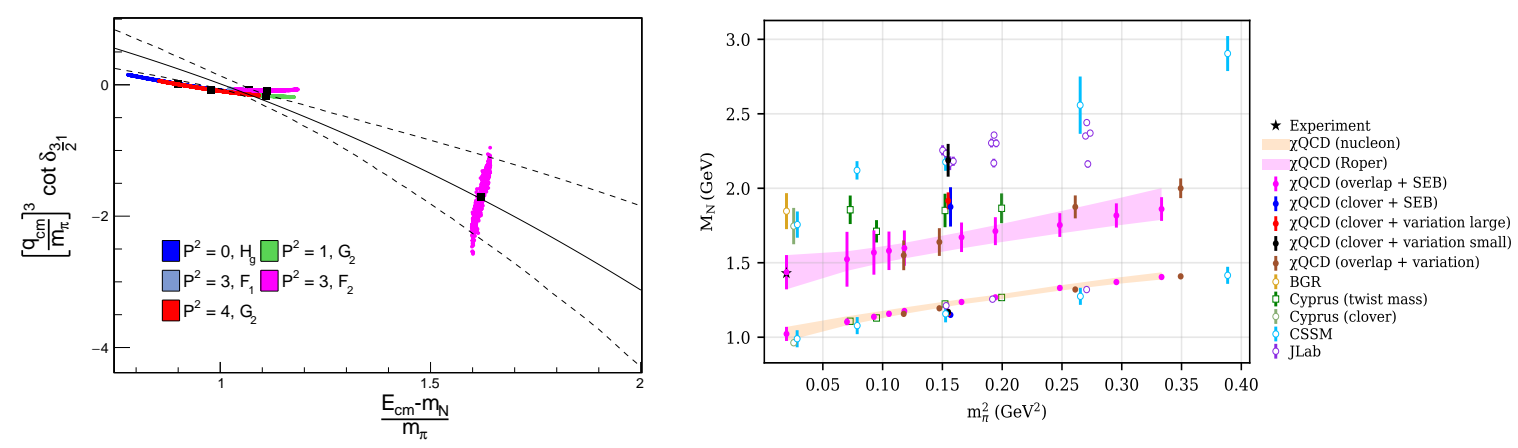

Figure 7: Left: (Figure adapted from Ref. [114]) $\frac{q_{c m}{ }^{3}}{m_{\pi}} \cot \delta_{\frac{3}{2}}$ as a function of the center of mass energy, $E_{c m}$ is shown for isospin-3/2 $N \pi$ scattering in $p$-wave. The $\Delta$ baryon appears in this channel. $E_{c m}$ is shown in the horizontal axis as a $\left(E_{c m}-m_{N}\right) / m_{\pi}$, where $m_{N}$ and $m_{\pi}$ are masses of nucleon and pion. In these units, the interval $(1,2)$ is elastic in the $N \pi$ scattering. Right: (Figure adapted from Ref. [112]) A summary of mass estimates for the nucleon and its first radial excitation from different lattice calculations.

$\Delta(1232)$ baryon is the lowest baryon resonance decaying to $N \pi$ in $p$-wave with branching fraction of $\sim 99.4 \%$ and a width $\sim 100 \mathrm{MeV}$. This is a good baryon candidate to be studied within an elastic approximation. All early calculations follow the single hadron approach. This is justified 
as in the ensembles with larger pion mass and smaller physical volumes like those used in these calculations, this baryon becomes a bound state. However, close to the physical pion mass and on large physical volumes determination of its resonance parameters requires a rigorous finite volume treatment. In Ref. [114], the authors perform a finite volume analysis to extract the $\Delta(1232)$ baryon resonance parameters in their lattice setup. Utilizing a single lattice QCD ensemble (CLS) with $N_{f}=2+1$ dynamical flavors of Wilson clover fermions with $m_{\pi}=280 \mathrm{MeV}$ and physical lattice size $L=3.67 \mathrm{fm}$, they observe the mass of the $\Delta(1232)$ baryon to be close to the $N \pi$ threshold. They find a good description of the resultant scattering amplitude using a Breit-Wigner shape with $m_{\Delta}=1344(20) \mathrm{MeV}$ and $g_{\Delta N \pi}^{B W}=19.0(4.7)$. In the left of Fig. 7, I present the figure adapted from Ref. [114], showing the phase shift as a function of the center of mass energy.

The Roper resonance (N(1440)) is another interesting low lying light baryon resonance. It was postulated by L. D. Roper to describe the $N \pi$ scattering data below $1.7 \mathrm{GeV}$ [115]. It is the first radial excitation of the nucleon, observed in two hadronic final states, $N \pi$ and $N \pi \pi$ and has a total width of $350 \mathrm{MeV}$. The $N \pi \pi$ final states could be arising from $N \eta, \Delta \pi$ and $N \sigma$. This indicates the need for a coupled channel finite volume analysis, possibly including three hadron interpolators, to discern the Roper resonance from the lattice. In the right of Fig. 7, I present the lattice results following single hadron approach for the ground and first excited state of the nucleon as a function of the pion mass squared (see Ref. [112] for a review). It is evident that all lattice calculations yield an expected nucleon mass that extrapolates to the physical value in the chiral limit. However, the first excitation is consistently found to be close to or above $1.7 \mathrm{GeV}$ even in the chiral limit, with the exception of calculations performed using chiral fermions [116, 117]. Only one investigation used five quark interpolators [118]. However, no levels in the energy interval $(1.2,1.8) \mathrm{GeV}$ are observed, including those levels related to expected scattering channels that are inevitable in the theory. Discussions on the disagreement between results from chiral and non-chiral actions are made in parallel talks during this meeting. Note that none of these calculations perform a rigorous finite volume treatment. Furthermore, no calculation finds any low lying levels in the energy region $(1.2,1.8) \mathrm{GeV}$ except for those using chiral fermions. The low lying first excitations in the chiral fermion calculations could be related to one of the expected scattering levels that are inevitable in the theory.

In this regard, Ref. [119] reports on an attempt to determine the excited spectrum of nucleon, including the scattering levels, and perform an elastic phase shift analysis within the Roper resonance energy region. This calculation studies only the spectrum in the rest frame of nucleon and has been performed only on a single PACS-CS ensemble with $N_{f}=2+1$ dynamical flavors of Wilson clover fermions with $m_{\pi}=156 \mathrm{MeV}$ and physical lattice size $L=2.9 \mathrm{fm}$. In a comparative study between the numerical results and theoretical expectation based on Lüscher's finite volume method, they find the energy levels in the spectrum to be consistent with the expected non-interacting level positions (see Fig. 8). This indicates the low lying Roper resonance does not arise on the lattice from the elastic $N \pi$ scattering. They also find signatures in operator state overlaps indicating strong coupled channel effects like those discussed in Ref. [118, 120], where the Roper resonance was described as a dynamically generated resonance due to coupled channel effects between $N \pi, N \sigma$ and $\Delta \pi$. This indeed calls for a more rigorous coupled channel investigation to understand the Roper resonance. Another interesting direction to pursue is to investigate the role of chiral symmetry in excited nucleon spectrum, considering the fact that the only calculations using chiral fermions give 

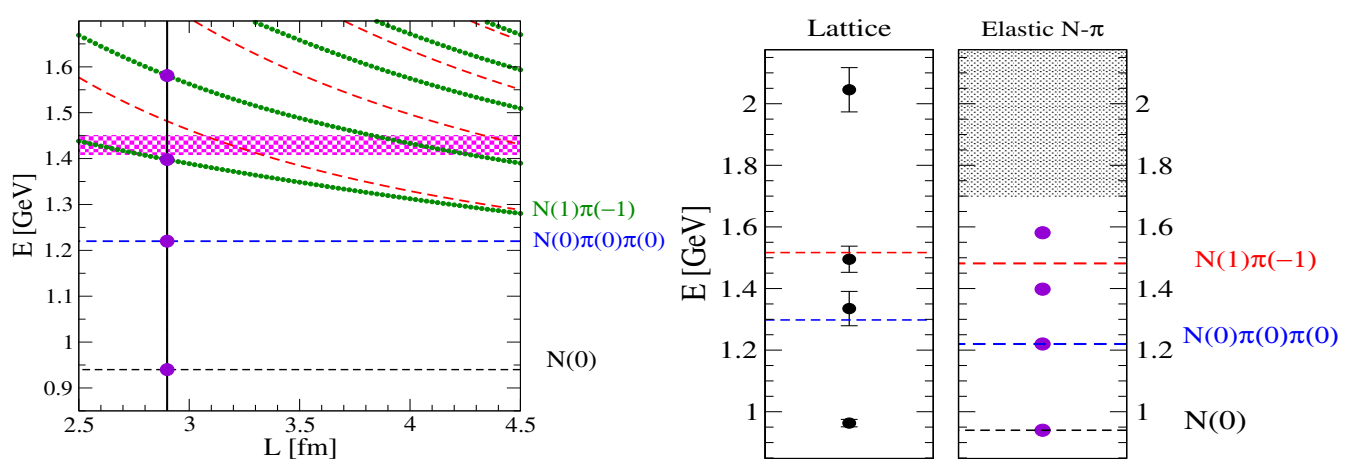

Figure 8: Figures adapted from Ref. [119]. Left: Excited energy spectrum of nucleons as a function of lattice size obtained by solving the inverse Lüscher finite volume problem for $m_{\pi}=156 \mathrm{MeV}$. Black, blue and red dashed lines indicate the non-interacting level positions for nucleon, $N \pi \pi$ and $N \pi$ levels, respectively. Dotted green lines indicate the $N \pi$ level positions levels in the presence of a Roper-like resonance coupled to them on the lattice. Right: Comparison of the excited nucleon spectrum between analytical expectations with numerical results from a lattice calculation with a pion mass $156 \mathrm{MeV}$ and physical spatial extension $2.9 \mathrm{fm}$.

low lying first excitations of the nucleon in the energy regime of the Roper resonance.

\section{Beyond mesons and baryons}

Many resonance structures, generally referred to as XYZs, have been discovered in the heavy quarkonium energy regime with properties contradicting the expectations from simple theoretical models. Starting with the discovery of $X(3872)$ in $B^{ \pm} \rightarrow K^{ \pm} X\left(X \rightarrow J / \psi \pi^{+} \pi^{-}\right)$decays by Belle in 2003 [121], currently there are several such candidates with an ambiguous nature in the charm and in the bottom sectors. Recently there has also been observation of baryons in the $\Lambda_{b}^{0} \rightarrow J / \psi K^{-} p$ decays by $\mathrm{LHCb}$ [7], that are interpreted as charmonium-nucleon pentaquarks. A recent summary of efforts to find theoretical description of these states can be found in Refs. [4, 5, 6].

Charmed tetraquarks : Early lattice calculations assuming elastic $D \bar{D}$ scattering extracted a bound state pole and argued it to be the lattice candidate for $X(3872)$ [122, 123]. In these calculations, the lattice levels are associated with non-interacting levels considering their nearness with the respective non-interacting level positions and the operator state overlaps. These studies followed a strategy of associating any additional energy level in the interacting spectrum, beyond those expected in the non-interacting spectrum, to indicate the presence of a narrow resonance. Alternatively, the deficiency of such additional levels is argued to indicate the absence any resonance, e.g. in the case of hidden charm $I=1$ sector [124]. Recently HSC has performed a detailed calculation to extract the finite volume spectra in the rest frame for $I=1$ hidden charm as well as doubly charm sectors using large bases of meson-meson and tetraquark interpolators [125]. Similar to other calculations referred to above, this calculation also followed the strategy of associating the extracted lattice energy levels with the expected non-interacting meson-meson energy levels in the region considered. They also do not find any strong signatures for presence of bound states or narrow resonances in the channels studied. All of these lattice calculations have been limited to zero momentum. It is argued in Ref. [123] that the tetraquark interpolators utilized are related to the 
meson-meson interpolators via Fierz relations [126] and interpreting lattice levels based on their overlaps with tetraquark interpolators is subtle. In a recent letter by the HAL QCD Collaboration using their finite volume formalism they investigate the interactions between $\pi J / \psi, \rho \eta_{c}$ and $\bar{D} D^{*}$ and argue that the charged $Z_{c}(3900)$ as a threshold cusp [127].

Doubly bottom tetraquarks : The existence of stable doubly heavy tetraquark states has been proposed using potential model calculations [128, 129] and heavy quark symmetry [130], for sufficiently large heavy quark mass. These calculations rely on the large mass of the heavy quark and so doubly bottom four quark systems are perhaps more interesting than doubly charm systems. This has motivated many lattice groups to perform investigations of these systems [131, 132, 133]. Calculations in Ref. [131] proceed by computing the potential of two static quarks in the presence of two light quarks, followed by solving the Schrödinger equation within BornOppenheimer approximation to study existing $\bar{b} \bar{b} q q$ states. In the $I\left(J^{P}\right)=0\left(1^{+}\right)$channel, they find a bound state $\Delta E=90_{-36}^{+43}$ below the $B B^{*}$ threshold. An extension of this work reported the existence of a tetraquark resonance for $l=1$, decaying into two B mesons, with quantum numbers $I\left(J^{P}\right)=0\left(1^{-}\right)$[132]. In Ref. [133], the authors study the axialvector channel for $I=1(\bar{b} \bar{b} u d)$ and $I=1 / 2(\bar{b} \bar{b} s u)$ using non-relativistic QCD for the bottom quarks and find unambiguous signals for deeply bound doubly bottom tetraquarks with binding energies 189(10) MeV and 98(7) MeV, respectively (see the left of Fig. 9). Recently the ILGTI Collaboration has also reported a similar calculation to investigate the light quark mass dependence of these binding energies [134] arriving at similar conclusions. The plot in the right of Fig. 9 shows the binding energy as a function of $m_{\pi}$ for $J^{P}=1^{+}$doubly bottom tetraquarks with different flavor contents.
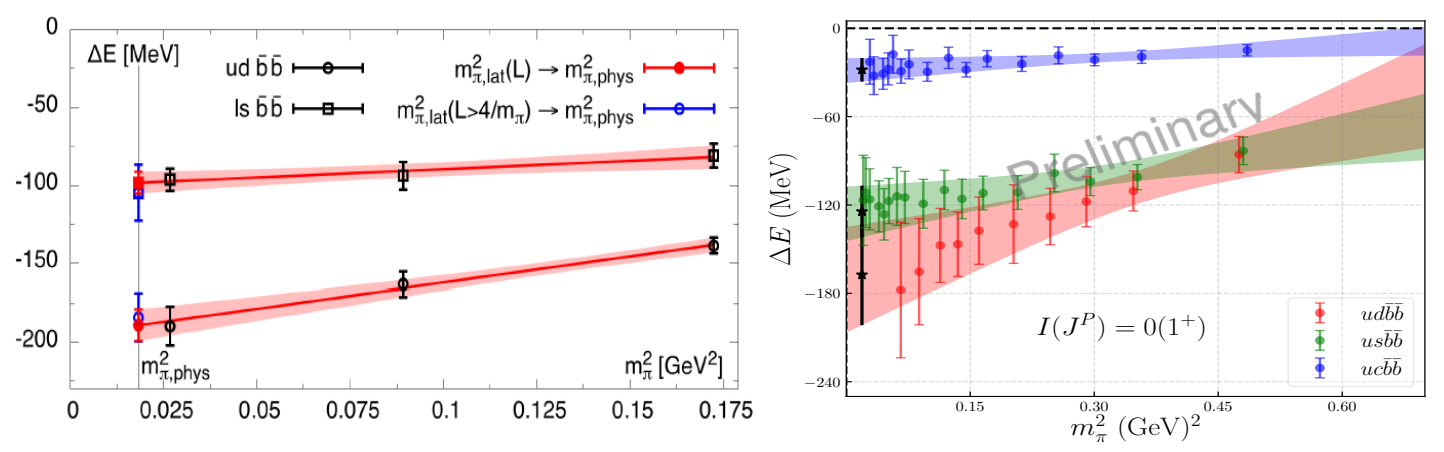

Figure 9: Results for binding energies of doubly bottom axialvector tetraquarks with different flavor contents as a function of $m_{\pi}^{2}$ from Ref. [133] (left) and Ref. [134] (right).

Penta quark systems : Investigations by NPLQCD have provided interesting evidence for a shallow bound state in $\eta_{c} N$ system at the $S U$ (3) flavor symmetric point [136]. Recent studies of the effects of the light hadron cloud (with pion mass as low as $223 \mathrm{MeV}$ ) on the potential between a static quark-antiquark pair have indicated many of these systems are energetically favorable with binding energies of less than a few $\mathrm{MeV}$ [137]. Preliminary results for elastic $J / \psi-N$ scattering are reported at this meeting [138]. Motivated by the recent discovery of two pentaquark candidates with spin 3/2 and 5/2 with opposite parities [7], studies are performed to extract the finite volume spectrum of the charmonium-nucleon system in the rest frame for both the parities. In Ref. [138], the authors perform lattice QCD study of $J / \psi-N$ and $\eta_{c}-N$ systems using HAL QCD finite 


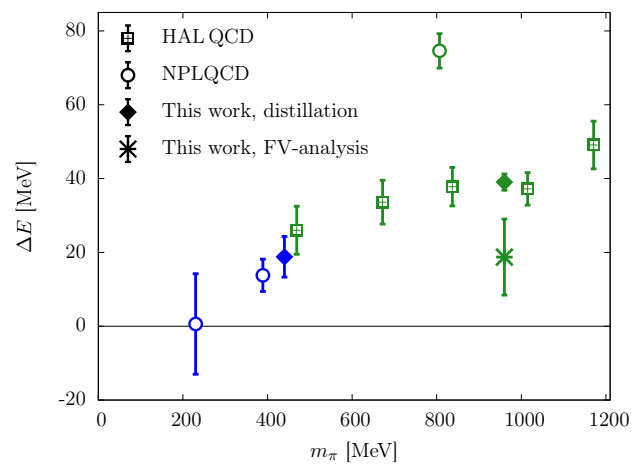

Figure 10: (Figure adapted from Ref. [135]) Comparison of binding energy of $H$-dibaryon between different lattice calculations. Green and blue colors refer to calculations at the SU(3) symmetric point and the SU(3) broken cases.

volume method and find these channels to be weakly attractive, although the attraction is not strong enough to have a bound state.

Six quark systems : Several contributions at this meeting discuss recent lattice calculations exploring baryon-baryon interactions. The HAL QCD Collaboration reports on the recent updates of their investigations of $N \Omega$ interactions at near physical pion mass [139]. In the $s$-wave spin 2 channel, from a $N_{f}=2+1$ flavor large volume lattice QCD simulation they find the possibility for a shallow quasi-bound state in the $N \Omega$ channel. In another recent article, HAL QCD report their study of $\Omega \Omega$ interactions resulting in shallow binding energies [140]. In Ref. [141], lattice group in Mainz report on their recent studies to resolve the question on whether uuddss ( $H$ dibaryon) system [142] is bound or not. There exists other lattice calculations performed in this regard by NPLQCD and HALQCD. All these early calculations reported a bound $H$ dibaryon at heavier than physical pion masses. In Ref. [141], the authors perform a $N_{f}=2$ flavor calculation using baryon-baryon interpolators as well as hexaquark operators. Performing a finite volume analysis á la Lüscher, they find a bound $H$ dibaryon with a binding energy $\Delta E=19 \pm 10 \mathrm{MeV}$ for a pion mass of $960 \mathrm{MeV}$. Comparison of various lattice results are made in Fig. 10.

\section{Summary}

Precision measurements of ground state hadrons are now well established using lattice QCD. Several calculations are quite successful in precisely postdicting/predicting the masses of ground state hadrons, composed of $u, d, s, c$ and $b$ quarks, that are well below the strong decay threshold. A summary of lattice calculations of the ground state baryons can be found in Fig. 1 and Fig. 2. Notably the mass of the recently discovered doubly charm baryon $\Xi_{c c}$ is in very good agreement with all the existing lattice predictions, demonstrating the ability of lattice QCD techniques to make reliable predictions. Exploratory calculations of excited baryon spectroscopy have been performed in a number publications in the past 10 years. The masses and quantum numbers of the recently discovered tower of $\Omega_{c}$ resonances by the LHCb Collaboration have been successfully predicted by such lattice investigations of excited $\Omega_{c}$ baryon spectrum. 
Several lattice calculations have been reported of elastic scattering of spinless particles to study the simplest hadronic resonances in the light, strange and charm meson sectors. Often most hadrons can several decay channels including scattering particles that have non-zero spin. Most of them are also open to 3-particle scattering channels, for which there is as yet no complete formalism that relates the three body scattering amplitudes with the discrete spectrum in a box. However, there is significant progress towards developing such a formalism. Calculations of coupled 2-particle scattering channels have been performed, where the pole singularities describing the scattering amplitudes are extracted by parameterizing the scattering matrix of these coupled channels. Recently there has also been a calculation, considering the scattering of mesons with non-zero spin. First calculation of a finite volume analysis of the $\Delta$ baryon and the Roper resonance are also reported in this review.

Motivated by experimental evidences for the existence of tetraquarks in the hidden charm sector, I reviewed some of the recent lattice calculations including the relevant meson-meson interpolators as well as tetraquark interpolators. Recent lattice investigations of the ground state doubly bottom four quark systems, inspired by phenomenological predictions, indicate deeply bound tetraquark states in the axialvector channels. Lattice calculations of hadron interactions in the $J / \psi-N$ channel and dibaryon systems were also briefly reviewed.

Continuing lattice QCD efforts, to study the hadron spectrum on several volumes including all relevant multi-hadron interpolators and to perform a rigorous finite volume analysis, are necessary to further understand these resonances. Considering the recent progress in hadron spectroscopy using lattice QCD calculations, a time when we could understand the experimentally observed hadron resonance structures directly from Quantum ChromoDynamics is not very far.

\section{Acknowledgments}

I thank R. Briceño, J. Bulava, S. Cali’, J. Dudek, G. Endrodi, K.F. Liu, A. T. Lytle, A. Palasseri, S. Paul, G. Rendón, U. Skerbis, F. Stokes, A. Veerappan and H. Wittig for sharing information and valuable inputs to this review. I am grateful to G. S. Bali, S. Collins, R. G. Edwards, P. Junnarkar, C. B. Lang, L. Leskovec, N. Mathur, D. Mohler, S. Mondal, M. Peardon, S. Piemonte, S. Prelovsek, A. Schäfer and S. Weishäpl for the pleasure in collaborating on various topics related to this review. In particular, I thank S. Collins and N. Mathur for various discussions in relation with this review. I acknowledge support from the EU under grant no. MSCA-IF-EF-ST-744659 (XQCDBaryons) and the Deutsche Forschungsgemeinschaft under grant No. SFB/TRR 55. I apologize for skipping some of the interesting calculations presented in this meeting due to time constraints.

\section{References}

[1] LHCB collaboration, R. Aaij et al., , Phys. Rev. Lett. 119 (2017) 112001 [1 707.01621 ].

[2] LHCb collaboration, R. Aaij et al., , Phys. Rev. Lett. 118 (2017) 182001 [1703. 04639 ].

[3] Belle collaboration, J. Yelton et al., , Phys. Rev. D97 (2018) 051102 [1711.07927].

[4] R. F. Lebed et al., Prog. Part. Nucl. Phys. 93 (2017) 143 [1610. 04528 ].

[5] A. Esposito, A. Pilloni and A. D. Polosa, Phys. Rept. 668 (2016) 1 [1611. 07920 ]. 
[6] S. L. Olsen, T. Skwarnicki and D. Zieminska, Rev. Mod. Phys. 90 (2018) 015003 [1 708.04012 ].

[7] LHCB collaboration, R. Aaij et al., , Phys. Rev. Lett. 115 (2015) 072001 [1507 . 03414 ].

[8] Belle collaboration, J. Yelton et al., , Phys. Rev. Lett. 121 (2018) 052003 [1805. 09384 ].

[9] LHCB collaboration, R. Aaij et al., , 1807.01919.

[10] LHCB collaboration, R. Aaij et al., , Phys. Rev. Lett. 121 (2018) 052002 [1806. 02744 ].

[11] C. Aubin et al., Phys. Rev. D70 (2004) 094505 [hep-lat/ 0402030 ].

[12] S. Durr et al., Science 322 (2008) 1224 [0 906 . 3599].

[13] MILC collaboration, A. Bazavov et al., , Rev. Mod. Phys. 82 (2010) 1349 [0903. 3598 ].

[14] R. J. Dowdall et al., Phys. Rev. D86 (2012) 094510 [1207. 5149 ].

[15] PACS-CS collaboration, Y. Namekawa et al., , Phys. Rev. D87 (2013) 094512 [1301.4743].

[16] S. Borsanyi et al., Science 347 (2015) 1452 [1406.4088].

[17] C. Liu, PoS LATTICE2016 (2017) 006 [1612 . 00103 ].

[18] R. A. Briceno et al., Rev. Mod. Phys. 90 (2018) 025001 [1 706.06223 ].

[19] LHP collaboration, S. Basak et al., , Phys. Rev. D72 (2005) 074501 [hep-lat/ 0508018 ].

[20] S. Basak et al., Phys. Rev. D72 (2005) 094506 [hep-lat/ 0506029 ].

[21] J. J. Dudek et al., Phys. Rev. D82 (2010) 034508 [1004 . 4930].

[22] C. E. Thomas et al., Phys. Rev. D85 (2012) 014507 [1107. 1930 ].

[23] C. Michael, Nucl. Phys. B 259 (1985) 58.

[24] M. Luscher, Commun. Math. Phys. 104 (1986) 177.

[25] B. Blossier et al., JHEP 0904 (2009) 094 [0 902 . 12 65].

[26] L. Maiani and M. Testa, Phys. Lett. B245 (1990) 585.

[27] M. Luscher, Commun. Math. Phys. 105 (1986) 153.

[28] M. Luscher, Nucl. Phys. B354 (1991) 531.

[29] M. Luscher, Nucl. Phys. B364 (1991) 237.

[30] R. A. Briceno, Phys. Rev. D89 (2014) 074507 [1 401 . 3312].

[31] R. A. Briceño, EPJ Web Conf. 175 (2018) 01016.

[32] T. D. Blanton et al., in Lattice 2018, East Lansing, US, July 22 - July 28, 2018, 1810.06634.

[33] M. Mai, in Proceedings, Lattice 2018: East Lansing, MI, US, July 22-28, 2018, 1810.00604.

[34] N. Ishii et al., Phys. Rev. Lett. 99 (2007) 022001 [nucl-th / 0611096 ].

[35] HAL QCD collaboration, N. Ishii et al., , Phys. Lett. B712 (2012) 437 [1203 . 3642 ].

[36] J. M. M. Hall et al., Phys. Rev. D85 (2012) 094502 [1201. 6114].

[37] D. Agadjanov et al., JHEP 06 (2016) 043 [1603. 07205].

[38] H.-W. Hammer et al., JHEP 09 (2017) 109 [1 706 . 07700].

[39] H. W. Hammer et al., JHEP 10 (2017) 115 [1707 . 02176]. 
[40] M. Doering et al., Phys. Rev. D97 (2018) 114508 [1802 . 03362].

[41] M. Mai and M. Doring, 1807.04746.

[42] S. Prelovsek, PoS LATTICE2014 (2014) 015 [1411. 0405 ].

[43] HS collaboration, M. Peardon et al., , Phys. Rev. D80 (2009) 054506 [0 905 . 2160 ].

[44] C. Morningstar et al., Phys. Rev. D83 (2011) 114505 [1104.3870].

[45] G. S. Bali et al., Comput. Phys. Commun. 181 (2010) 1570 [0 910 . 3970].

[46] C. Alexandrou and C. Kallidonis, Phys. Rev. D96 (2017) 034511 [1704.02647].

[47] C. Alexandrou et al., Phys. Rev. D90 (2014) 074501 [1406.4310].

[48] PACS-CS collaboration, S. Aoki et al., , Phys. Rev. D79 (2009) 034503 [0 807.1661 ].

[49] W. Bietenholz et al., Phys. Rev. D84 (2011) 054509 [1102.5300].

[50] A. Portelli, PoS LATTICE2014 (2015) 013 [1505 . 070 57].

[51] J. Bulava et al., Phys. Rev. D82 (2010) 014507 [1 004 . 5072 ].

[52] R. G. Edwards et al., Phys. Rev. D84 (2011) 074508 [11 04 . 5152].

[53] HS collaboration, R. G. Edwards et al., , Phys. Rev. D87 (2013) 054506 [1212 . 5236 ].

[54] BGR collaboration, G. P. Engel et al., , Phys. Rev. D87 (2013) 074504 [1301.4318].

[55] S. e. a. Basak, PoS LATTICE2012 (2012) 141 [1211. 6277].

[56] S. Basak et al., PoS LATTICE2013 (2014) 243 [1312 . 3050 ].

[57] ILGTI collaboration, S. Basak et al., , PoS LATTICE2014 (2015) 083 [1412 . 7248 ].

[58] N. Mathur and M. Padmanath, 1807.00174.

[59] R. A. Briceno et al., Phys. Rev. D86 (2012) 094504 [1207. 3536].

[60] Z. S. Brown et al., Phys. Rev. D90 (2014) 094507 [1 409.0497 ].

[61] P. Pérez-Rubio et al., Phys. Rev. D92 (2015) 034504 [1503. 08440 ].

[62] TWQCD collaboration, Y.-C. Chen and T.-W. Chiu, , Phys. Lett. B767 (2017) 193 [1701. 02581$].$

[63] S. Durr et al., Phys. Rev. D86 (2012) 114514 [1208. 6270].

[64] M. Padmanath and N. Mathur, in Proceedings, CHARM 2015: Detroit, USA, May 18-22, 2015, $2015,1508.07168$.

[65] N. Mathur et al., 1806.04151.

[66] R. Lewis et al., Phys. Rev. D64 (2001) 094509 [hep-ph/ 0107037 ].

[67] N. Mathur et al., Phys. Rev. D66 (2002) 014502 [hep-ph/ 0203253 ].

[68] UKQCD collaboration, J. M. Flynn et al., , JHEP 07 (2003) 066 [hep-lat / 0307025 ].

[69] L. Liu et al., Phys. Rev. D81 (2010) 094505 [0909.3294].

[70] SELEX collaboration, M. Mattson et al., , Phys. Rev. Lett. 89 (2002) 112001 [hep-ex/0208014].

[71] X. Liao and T. Manke, hep-lat/0210030.

[72] J. J. Dudek et al., Phys. Rev. D77 (2008) 034501 [0707.4162]. 
[73] J. J. Dudek et al., Phys. Rev. Lett. 103 (2009) 262001 [0909. 0200].

[74] HS collaboration, L. Liu et al., , JHEP 07 (2012) 126 [12 04 . 542 5].

[75] G. Moir et al., JHEP 05 (2013) 021 [1301 . 7670].

[76] HS collaboration, G. K. C. Cheung et al., , JHEP 12 (2016) 089 [1610 . 01073 ].

[77] S. Collins et al., $1811 . \times x \times x \times$.

[78] G. S. Bali et al., Phys. Rev. D84 (2011) 094506 [1110.2381].

[79] D. Mohler et al., Phys. Rev. D87 (2013) 034501 [1208. 4059].

[80] M. Wurtz et al., Phys. Rev. D92 (2015) 054504 [1505.04410].

[81] M. Padmanath and N. Mathur, Phys. Rev. Lett. 119 (2017) 042001 [1 704.00259 ].

[82] J. M. Bulava et al., Phys. Rev. D79 (2009) 034505 [0 901 . 0027 ].

[83] M. Padmanath et al., Phys. Rev. D90 (2014) 074504 [1307 . 7022 ].

[84] M. Padmanath et al., Phys. Rev. D91 (2015) 094502 [1502.01845].

[85] S. Meinel, Phys. Rev. D85 (2012) 114510 [1202.1312].

[86] M. Padmanath et al., PoS LATTICE2014 (2015) 084 [1410. 8791].

[87] M. Padmanath et al., in Proceedings, Charm 2013: Manchester, UK, August 31-September 4, 2013, 2013, 1311.4806.

[88] CP-PACS collaboration, S. Aoki et al., , Phys. Rev. D76 (2007) 094506 [0 708 . 3705 ].

[89] ETM collaboration, K. Jansen et al., , Phys. Rev. D80 (2009) 054510 [0 906 . 4720].

[90] CS collaboration, S. Aoki et al., , Phys. Rev. D84 (2011) 094505 [1106.5365].

[91] C. B. Lang et al., Phys. Rev. D84 (2011) 054503 [1105. 5636$].$

[92] C. Pelissier and A. Alexandru, Phys. Rev. D87 (2013) 014503 [1211. 0092 ].

[93] HS collaboration, J. J. Dudek et al., , Phys. Rev. D87 (2013) 034505 [1212 . 0830 ].

[94] D. J. Wilson et al., Phys. Rev. D92 (2015) 094502 [1 507.02599 ].

[95] RQCD collaboration, G. S. Bali et al., , Phys. Rev. D93 (2016) 054509 [1512 . 08678 ].

[96] D. Guo et al., Phys. Rev. D94 (2016) 034501 [1605.03993].

[97] Z. Fu and L. Wang, Phys. Rev. D94 (2016) 034505 [1608 . 07478].

[98] C. Alexandrou et al., Phys. Rev. D96 (2017) 034525 [1 704.05439 ].

[99] S. Prelovsek et al., Phys. Rev. D88 (2013) 054508 [1307. 0736 ].

[100] D. J. Wilson et al., Phys. Rev. D91 (2015) 054008 [1411. 2004 ].

[101] HS collaboration, J. J. Dudek et al., , Phys. Rev. Lett. 113 (2014) 182001 [1406.4158].

[102] R. Brett et al., Nucl. Phys. B932 (2018) 29 [1802 . 03100 ].

[103] R. A. Briceno et al., Phys. Rev. D97 (2018) 054513 [1708. 06667 ].

[104] HS collaboration, J. J. Dudek et al., , Phys. Rev. D88 (2013) 094505 [1309. 260 8].

[105] R. A. Briceno et al., Phys. Rev. Lett. 118 (2017) 022002 [1607. 05900 ]. 
[106] D. Guo et al., Phys. Rev. D98 (2018) 014507 [1803. 02897 ].

[107] A. Woss et al., JHEP 07 (2018) 043 [1802 . 05580].

[108] G. S. Bali et al., Phys. Rev. D96 (2017) 074501 [1706.01247].

[109] D. Mohler et al., Phys. Rev. Lett. 111 (2013) 222001 [1308. 3175].

[110] Belle collaboration, K. Chilikin et al., , Phys. Rev. D95 (2017) 112003 [1704. 01872 ].

[111] C. B. Lang et al., JHEP 09 (2015) 089 [1 503 . 05363].

[112] K.-F. Liu, Int. J. Mod. Phys. E26 (2017) 1740016 [1609. 02572].

[113] C. B. Lang and V. Verduci, Phys. Rev. D87 (2013) 054502 [1212 . 5055].

[114] C. W. Andersen et al., Phys. Rev. D97 (2018) 014506 [1710. 01557].

[115] L. D. Roper, Phys. Rev. Lett. 12 (1964) 340.

[116] K.-F. Liu et al., PoS LATTICE2013 (2014) 507 [1 403 . 6847 ].

[117] N. Mathur et al., Phys. Lett. B605 (2005) 137 [hep-ph/ 0306199 ].

[118] A. L. Kiratidis et al., Phys. Rev. D95 (2017) 074507 [1608.03051].

[119] C. B. Lang et al., Phys. Rev. D95 (2017) 014510 [1610.01422].

[120] J.-j. Wu et al., Phys. Rev. D97 (2018) 094509 [1703.10715].

[121] Belle collaboration, S. K. Choi et al., , Phys. Rev. Lett. 91 (2003) 262001 [hep-ex/ 0309032 ].

[122] S. Prelovsek and L. Leskovec, Phys. Rev. Lett. 111 (2013) 192001 [1307. 5172].

[123] M. Padmanath et al., Phys. Rev. D92 (2015) 034501 [1503.03257].

[124] S. Prelovsek and L. Leskovec, Phys. Lett. B727 (2013) 172 [1308 . 2097].

[125] HS collaboration, G. K. C. Cheung et al., , JHEP 11 (2017) 033 [1709.01417].

[126] J. F. Nieves and P. B. Pal, Am. J. Phys. 72 (2004) 1100 [hep-ph/ 0306087$].$

[127] HAL QCD collaboration, Y. Ikeda et al., , Phys. Rev. Lett. 117 (2016) 242001 [1602. 03465$].$

[128] J. Carlson et al., Phys. Rev. D37 (1988) 744.

[129] M. Karliner and J. L. Rosner, Phys. Rev. Lett. 119 (2017) 202001 [1707. 07666 ].

[130] E. J. Eichten and C. Quigg, Phys. Rev. Lett. 119 (2017) 202002 [1 707.09575$].$

[131] P. Bicudo et al., Phys. Rev. D95 (2017) 034502 [1612. 02758].

[132] P. Bicudo et al., Phys. Rev. D96 (2017) 054510 [1704. 02383 ].

[133] A. Francis et al., Phys. Rev. Lett. 118 (2017) 142001 [1607.05214].

[134] P. Junnarkar et al., EPJ Web Conf. 175 (2018) 05014 [1712. 08400 ].

[135] A. Hanlon et al., 2018, 1810.13282.

[136] S. R. Beane et al., Phys. Rev. D91 (2015) 114503 [1410. 7069 ].

[137] M. Alberti et al., Phys. Rev. D95 (2017) 074501 [1608. 06537].

[138] T. Sugiura et al., EPJ Web Conf. 175 (2018) 05011 [1711.11219].

[139] T. Iritani et al., 1810.03416.

[140] S. Gongyo et al., Phys. Rev. Lett. 120 (2018) 212001 [1709. 00654 ].

[141] A. Francis et al., 1805.03966.

[142] R. L. Jaffe, Phys. Rev. Lett. 38 (1977) 195. 